Carlos Enrique Cabrera-Pivaral 1,2 Centeno Lopez Ninel Mayari 1,2 Jose Miguel Arredondo Trueba ${ }^{1}$ Guillermo Julian Gonzalez Perez 1

Maria Guadalupe Vega Lopez 1

Isabel Valadez Figueroa 1

Maria Guadalupe Aldrete Rodriguez 1

\section{Evaluación de dos estrategias de educación nutricional vía radio en Guadalajara, México}

\author{
Evaluation of two strategies for nutritional \\ education using radio programs \\ in Guadalajara, Mexico
}

1 Centro de Estudios en Salud, Población y Desarrollo, Centro Universitario en Ciencias de la Salud, Universidad de Guadalajara. Sierra Mojada 950, C. P. 44340, Centro Médico, Colonia Independencia, Guadalajara, Jalisco, México.

2 Unidad de Investigación Médica en Epidemiología Clínica, Hospital de Especialidades, Centro Médico Nacional de Occidente, Instituto Mexicano del Seguro Social. Belisario Dominguez 1000, C. P. 44340, Centro Médico, Colonia Independencia, Guadalajara, Jalisco, México.

\begin{abstract}
Modifying knowledge and attitudes through persuasive communication in health via radio has produced encouraging results for public health planners. This study's objective was to measure the effect of an educational strategy on knowledge and attitudes towards nutrition in two marginalized communities in Guadalajara, Mexico. Two communities were randomly selected. In each community a group of individuals was invited to be exposed to radio broadcasts. Using a coded and structured instrument, knowledge and attitudes towards the contents of nutritional education for health were measured before and after the intervention in both groups. Group A ( $n=37)$ was organized and exposed to the dynamics of the radio forum throughout the 4 months during which the project lasted. Group $B(n=33)$ was not organized, and listened to the radio program according to its own cultural dynamics. Median knowledge and attitudes (KA) for group $A$ was 56.8 in the pre-test and 74.1 in the post-test ( $W: p=-0.05$ ). In group $B$ the $K A$ results were 53.0 and 59.2, respectively ( $W: p=-0.05$ ). The results emphasize the advantages of the radio forum as a health communications strategy for human nutrition.
\end{abstract}

Key words Radio; Nutrition Education; Health Promotion

Resumen La modificación de los conocimientos y de las actitudes desde la óptica de la comunicación persuasiva en salud vía radio ha reportado resultados alentadores para los planificadores sanitarios. El objetivo fue medir el efecto de una estrategia educativa en los conocimientos y en las actitudes, utilizando el radio, en dos comunidades marginadas. Es una intervención educativa vía radio en dos comunidades marginadas y socioeconómicamente homogéneas en Guadalajara, Jalisco, México. Se seleccionaron al azar dos comunidades marginadas y se formaron dos grupos de radioescuchas, se midieron los conocimientos y las actitudes sobre contenidos de educación nutricional para la salud mediante un instrumento codificado y estructurado. El primer grupo "A", de 37 sujetos, fue organizado y expuesto a la dinámica del radio foro durante los 4 meses que dura el proyecto. El grupo " $B$ " ( $n=33)$, no organizado, se expuso al proyecto radiofónico según su propia dinámica cultural. La mediana del grupo A para Conocimientos y Actitudes (CA), en la pre medición, fue de 56,8 y, para la pos medición, fue de 74,1 (W: $p=-0,05)$. Para el grupo B, los CA fueron de 53 y 59,2, respectivamente ( $W: p=-0,05)$. Así se refuerzan las ventajas del radio foro como estrategia comunicativa en salud en el área de la nutrición humana.

Palabras clave Radio; Educación Nutricional; Promoción de la Salud 


\section{Introducción}

La utilización de los medios de comunicación en programas educativos de índole formal o informal, en países en desarrollo o altamente industrializados, ha reportado resultados que en mucho se apegan a lo esperado por los planificadores de esfuerzos educacionales.

En especial los electrónicos son los que, por su naturaleza misma, más han atraído la atención de quienes conciben e implementan proyectos encaminados a la enseñanza o al desarrollo comunitario; tanto el radio como la televisión paulatinamente se han venido incorporando a la vida cotidiana de la sociedad actual (Alva, 1995).

A pesar de que el radio y la televisión se han aprovechado en diversos campos educativos, aún hay bastante por explorar en cuanto a las bondades y limitaciones de dichos recursos de comunicación. Además, con el aporte de esos recursos, no todas las áreas del conocimiento y del desarrollo social se han tomado en cuenta como contenido central del aprendizaje individual y colectivo, tal es el caso de la salud (De Roux, 1994; Salleras, 1985).

Las posibilidades del radio para incrementar el conocimiento y modificar las actitudes como base de la educación sanitaria son el principal interés de este manuscrito, sobre todo al resaltar que este medio electrónico - único por sus alcances, costos y penetración - es utilizado en muy contadas veces, además de desconocerse su real y verdadera potencialidad, lo que ha acarreado el uso irracional y por debajo de su propia capacidad.

Para quienes se han preocupado por estudiar las posibilidades de la comunicación de masas con objetivos de cambio y desarrollo social, específicamente en aquellas regiones del mundo no altamente industrializadas o de bajo ingreso, de algún modo han ponderado las potencialidades del radio - medio electrónico de inigualable penetración - como elemento dinamizador de procesos educativos que sean susceptibles de conducir a una población en particular a incrementar su conocimiento de la realidad, a transformar y, a su vez, a ayudarla a asumir e implementar decisiones que contribuyan a la solución de un problema colectivo (Salleras, 1985).

Jamison \& Mcanany (1984), refiriéndose a las bondades del radio, señalan que, a pesar de los avances tecnológicos en el campo de las telecomunicaciones, el radio continuará siendo, al menos durante los próximos 10 años, el medio masivo más popular y extendido. Puesto que, en comparación con otros, el radio se muestra como el camino para alcanzar propósitos educacionales y de desarrollo, debido, principalmente, a sus costos no elevados y a las propiedades logísticas de relativa complejidad de los esfuerzos de entrenamiento técnico que requiere su uso.

En América Latina, el radio ha demostrado ser un apoyo importante en la generación de proyectos con base comunitaria que, en una última instancia, persiguen la búsqueda y la aplicación de mecanismos viables de participación y autogestión (Díaz, 1993).

\section{Material y métodos}

Tratase de una intervención comunicativaeducativa, y su propósito fue valorar la bondad de la estrategia comunicativa sobre los conocimientos (C) y las actitudes (A) acerca de la nutrición, en dos comunidades. La medición de $\mathrm{C}$ y A ha sido hecha antes y después del proyecto de intervención radiofónico.

Las comunidades - seleccionadas al azar de la zona conurbada de Guadalajara, Jalisco, México - se expusieron a la intervención comunicativa a través de un medio radiofónico con amplia penetración en las comunidades.

El grupo "A" se caracterizó por ser expuesto al "radio foro"; la exposición duró 20 minutos, le continuó una discusión de 40 minutos (en promedio), coordinada por un trabajador de la salud con previa capacitación. El grupo se integró a partir de una asamblea comunitaria y el radio foro se desarrolló en la escuela primaria de la propia localidad. La intervención radiofónica fue semanal durante un lapso de tiempo de 16 semanas. La medición inicial (10 minutos antes de la exposición), la medición final (15 minutos después de la exposición al foro de discusión) y la recolección de datos fueron cogidas mediante una encuesta estructurada y codificada del tipo "Likert", aplicada por un equipo de trabajadoras sociales adiestradas.

El grupo “B” se caracterizó por ser expuesto al "radio" con duración de 20 minutos. El grupo de radio escuchas se integró a partir de una visita inicial casa por casa y se logró el listado nominal. La exposición al medio fue efectuada en sus habitaciones. La intervención radiofónica fue semanal, durante el lapso de tiempo de 16 semanas. Las recolecciones inicial (10 minutos antes de la exposición) y final (15 minutos después de la exposición) de datos, así como su medición, fueron logradas mediante una encuesta estructurada y codificada tipo "Likert" aplicada por una trabajadora social adiestrada. 
En las comunidades (Grupos A y B), se midieron el Conocimiento, (se refiere a la información aprendida) y las Actitudes (se refieren a la intención manifiesta) (Salleras, 1985) sobre la nutrición. Los contenidos educativos de la intervención radiofónica, para ambos grupos "A" y "B", se referían a producción, distribución, disponibilidad, consumo, utilización biológica y eliminación de alimentos y nutrimentos.

La elaboración de guiones radiofónicos y de materiales de apoyo fue responsabilidad del equipo técnico y de especialistas en materia de comunicación y nutrición. El tema de la nutrición fue considerado útil por las familias de las comunidades y surge de un análisis de necesidades; se traduce al lenguaje de la radio bajo el nombre de "Dime lo que comes y te diré quien eres”. La historia es protagonizada por un adolescente que viaja de la ciudad a un pequeño rancho de su abuelo campesino. En el marco de situaciones y sucesos de la vida diaria en el campo, los personajes - a quienes a lo largo de la serie se van sumando otros - van tejiendo el contenido que toca diversos aspectos de producción, distribución, disponibilidad, consumo y utilización de los alimentos.

El instrumento para la medición de los conocimientos y de las actitudes, se integró de 24 preguntas (10 de conocimientos y 14 de actitudes), estructuradas tipo "Likert"; esa medición integró una calificación de 0 a 100 puntos. El análisis estadístico para comparar los dos grupos fue el estadígrafo de Wilcoxon, para grupos relacionados, con medición ordinal de antes y después, además de su variación respecto a algunos factores sociodemográficos (Siegel, 1986).

\section{Resultados}

\section{Datos sociodemográficos}

El grupo de experimento organizado como "radio foro" quedó constituido por 37 participantes. El $81 \%$ de ellos eran mujeres y el $51 \%$, casados. La mayoría reportó tener estudios incompletos de primaria (73\%), destacandose un $19 \%$ de personas que no sabían leer y escribir. Las ocupaciones principales registradas fueron: agricultura (21,6\%), oficios domésticos $(48,6 \%)$ y escolares $(16,2 \%)$. De los que informaron acerca de sus ingresos, el 45,9 por ciento tenía percepciones entre 2 y 3 salarios mínimos vigentes.

El grupo de control se constituyó de 33 participantes. El 75,7\% de ellos son mujeres. Las personas casadas alcanzaron el 54,5 por ciento. La mayoría reportó tener estudios incompletos de primaria $(69,7 \%)$, destacandose un
18,2 por ciento de personas que no sabían leer y escribir. Las ocupaciones principales registradas fueron: agricultura $(18,2 \%)$, oficios domésticos (51,5\%) y escolares (15,1\%). De los que informaron acerca de sus ingresos, el 51,5 por ciento tenía percepciones entre 2 y 3 salarios mínimos vigentes (Tabla 1).

\section{Emisiones radiofónicas}

Se efectuaron 12 emisiones radiofónicas a través de una radiodifusora local con adecuado nivel de popularidad en ambas comunidades. En cada grupo de estudio y para cada emisión, se midieron, antes y después, los conocimientos y las actitudes acerca de la nutrición humana.

La primera emisión radiofónica atendió al tema de los "Principios básicos de la nutrición humana"; el grupo "radio foro" logró una media de 44,5 en la pre medición, la que disminuyó después de escuchar el programa y participar del foro. Dicha disminución se debe a la falta de experiencia con el instrumento de medición y a la modalidad educativa participativa que confrontó el conocimiento previo. Esa dis-

\begin{tabular}{|c|c|c|c|c|}
\hline \multirow[t]{2}{*}{ Variable } & \multicolumn{2}{|c|}{ Grupo B (control) } & \multicolumn{2}{|c|}{ Grupo A (radio foro) } \\
\hline & $n$ & $\%$ & $\mathrm{n}$ & $\%$ \\
\hline \multicolumn{5}{|l|}{ Género } \\
\hline Mujer & 25 & 75,7 & 30 & 81,0 \\
\hline Hombre & 8 & 24,3 & 7 & 19,0 \\
\hline \multicolumn{5}{|l|}{ Estado civil } \\
\hline Casados & 18 & 54,5 & 19 & 51,4 \\
\hline Otros & 15 & 45,5 & 18 & 48,6 \\
\hline \multicolumn{5}{|l|}{ Escolaridad } \\
\hline No lee no escribe & 6 & 18,2 & 7 & 19 \\
\hline Primaria incompleta & 23 & 69,7 & 27 & 73,0 \\
\hline Primaria completa & 4 & 12,1 & 3 & 8,0 \\
\hline \multicolumn{5}{|l|}{ Ocupación } \\
\hline Agricultura & 6 & 18,2 & 8 & 21,6 \\
\hline Ama de casa & 17 & 51,5 & 18 & 48,6 \\
\hline Escolar & 5 & 15,1 & 6 & 16,2 \\
\hline Outro & 5 & 15,1 & 5 & 13,5 \\
\hline \multicolumn{5}{|l|}{ Ingreso } \\
\hline 2-3 salarios mínimos & 17 & 51,5 & 17 & 45,9 \\
\hline Menos de $2 \mathrm{smv}$ & 16 & 48,5 & 20 & 54,1 \\
\hline
\end{tabular}

Fuente: directa; $p>0,05$. 
minución no se compartió con el grupo control (Tabla 2).

La segunda audición, "La producción de alimentos", se muestra de mayor interés, lo que redunda en aumento de su calificación entre la audición previa y la posterior.

La tercera aborda aspectos sobre la "Distribución de los alimentos”, y ambos grupos mantienen un leve incremento de sus puntuaciones promedio.

En la cuarta emisión radiofónica, que abordó el tema de la "Disponibilidad de los alimentos”, el grupo control logró mejor promedio que el grupo experimental.

En la quinta emisión, se analizó el "Consumo de los alimentos", y el comportamiento de sus mediciones mostró una franca ventaja del grupo radio foro sobre el grupo control.

A partir de la sexta emisión radiofónica, "Utilización biológica de los alimentos”, el grupo radio foro reportó medias que van de una mínima de 80, comparativamente con el grupo de control, el cual, a pesar de sufrir leves incrementos, dista del promedio del grupo experimental.

La séptima y la octava audiciones introdujeron información propia de los nutrientes y de los distintos grupos de alimentos que se deben consumir en la dieta diaria, enfatizando los daños a la salud causados por una mala nutrición (desnutrición, obesidad, diabetes e hipertensión, entre otros).

Nombrar y explicar las llamadas etapas "críticas” en la vida del ser humano (lactancia, ado-

\section{Tabla 2}

Estrategia comunicativa en nutrición según grupo.

\begin{tabular}{lccccc}
\hline \multirow{2}{*}{ Número da Emisión } & \multicolumn{2}{c}{ Control } & \multicolumn{2}{c}{ Radio foro } \\
& pre & post & \multicolumn{2}{c}{ pre } & post \\
\hline 1 & 40 & 42 & 44,5 & 35 \\
2 & 43 & 45 & 46 & 53 \\
3 & 58 & 60 & 55 & 65 \\
4 & 55 & 63 & 50 & 58 \\
5 & 45 & 49 & 58 & 65 \\
6 & 60 & 65 & 65 & 80 \\
7 & 55 & 65 & 63 & 85 \\
8 & 65 & 60 & 60 & 90 \\
9 & 55 & 60 & 63 & 88 \\
10 & 60 & 68 & 58 & 95 \\
11 & 50 & 70 & 55 & 90 \\
12 & 50 & 65 & 65 & 85 \\
Mediana & 53 & 59,2 & 56,8 & 74,1 \\
D Estandar & 7,5 & 9,3 & 7,1 & 18 \\
\hline
\end{tabular}

Fuente: directa; Wilcoxon: $p \leq 0,05$. lescencia y vejez), así como recomendar dietas para esas edades, hacen los contenidos de la novena y de la décima audiciones los más interesantes. Ambos grupos logran incrementos, pero el radio foro muestra una mayor ventaja.

En la décima, se observa el mayor incremento de la serie, en favor del grupo radio foro que, de 58 en la pre medición, pasó a 95 en la post medición.

En la undécima audición, se concluye con los contenidos programados. Para esa edición, se elegieron las vitaminas y los minerales. Los resultados de las mediciones muestran que el grupo radio foro mantuvo su ventaja, a pesar de que el grupo control logró aquí el mejor incremento de la serie radiofónica.

Finalmente, en el espacio de la duodécima audición, se trabajó una síntesis o una recopilación de los contenidos vistos a lo largo de la serie, encontrando que, en las medias de los diferentes grupos, se mantiene la ventaja del radio foro.

Al relacionar el valor final logrado en las emisiones radiofónicas con las variables sociodemográficas estudiadas, no se observó diferencia estadística entre ambos grupos ( $p>0,05)$, exepto en la décima emisión, a favor del sexo femenino $(97,5 \pm 8,7$ vrs 92,3 $\pm 19,4$ del masculino con $\mathrm{p}=0,01$ ).

\section{Discusión}

En la ganancia de conocimientos y actitudes que se produjeron a través de la serie de 12 programas, el radio foro (W: $p=-0,05$ ), se concibe como un instrumento útil y eficaz, para apoyar o dinamizar esfuerzos de educación sanitaria (Rees, 1999). Siempre y cuando éste tenga como principal sustento el ámbito cultural, el compromiso y la voluntad de la comunidad y de las instituciones que lo avalen y lo legitimen (Steinfatt, 1986).

El tomar en cuenta la información que una comunidad tiene previo al inicio de un proyecto educativo permite señalar un punto de referencia importante, que puede intervenir en la dinámica del "radio foro" y del grupo "control" y puede evitar caer en información poco novedosa y reiterativa, que limitara su participación grupal (Núñez, 1989).

El género femenino fue él más representado (81\% y $75,7 \%$, para los grupos respectivos), sin embargo no mostró diferencias estadísticamente significativas en la medición final ( $\mathrm{p} \geq$ 0,05 ), a pesar de mostrar mediciones por arriba en ambos grupos. Sin duda ese proyecto radiofónico ocasiona un mayor grado de reflexión 
en dicho grupo ya que presupone una experiencia de vida, que apeló más a la conciencia, a las inquietudes y a los roles sociales (Solano, 1992).

En la cuarta y la quinta emisiones, la disponibilidad y la distribución de los alimentos fueron los puntos centrales. En ese programa se ventilaron algunos tópicos críticos que generan problemas al consumidor (Vgr. acaparamiento y alza de precios en productos básicos, el intermediario, etc.), lo que ocasionó que los comentarios, en el grupo radio foro, mostraran gran interés y motivación, lo que se reflejó en la medición y en la riqueza de la discusión - sobre todo en adultos hombres - y fue más allá de lo que esperaba suscitar mediante el libreto radiofónico (Bitzer, 1993).

Lo que acontece a partir de la octava audición con las evaluaciones de los distintos grupos, especialmente en el radio foro, pone de relieve las ventajas de un evento radiofónico sistematizado y continuo, que se vuelve familiar a los escuchas (Flora et al., 1989; Lingfors, 2001).

Las mujeres, aunque es el género más representado, no muestra una diferencia significativa con respecto al resto. Excepto en la décima emisión ( $\mathrm{W}=4,3 ; \mathrm{p}=0,01$ ) y en favor del grupo "radio foro". Recordando que ésta abordó contenidos sobre enfermedades que pueden afectar la nutrición de los niños, además de hablar de los padecimientos que perturban la asimilación de nutrientes en los ancianos y en los adultos.

Las reacciones que los receptores tuvieron ante distintos factores ajenos al teórico esquema lineal y unidireccional de la comunicación (Mercado, 1990) - el emisor (A) que utiliza un medio $(\mathrm{X})$ para hacer llegar algún mensaje $(\mathrm{Y})$ al oyente (B) nos lleva a subrayar el hecho de que los elementos participantes en todo proceso comunicacional no están aislados de una realidad manifiesta, en este caso denominada la "atmósfera" de la comunicación. Así mismo, al pensar en el aprovechamiento del radio como dinamizador o apoyo didáctico, no se puede olvidar su íntima dependencia con los otros medios - formales o no - y con el contexto sociocultural del grupo objetivo (Flora et al., 1989; Verhaegen, 1980).

Otras experiencias latinoamericanas (Rochon, 1991) y africanas con el radio foro reportan ganancias similares o mayores a las alcanzadas en el grupo de experimento de este reporte. Además, no está de sobra acotarlo, este recurso de comunicación posee la virtud de difundir - en el mismo espacio y tiempo - un mensaje de recepción grupal, lo cual motiva a que su contenido inicie el proceso de socializa- ción y de reafirmación más rápidamente que lo escuchado y descodificado individualmente, y además facilita la retroalimentación directa del oyente al emisor, planificador o guía de un esfuerzo educativo (Boyd et al., 1998; Rogers et al., 1999; Vaughan et al., 2000).

En Latinoamérica, la existencia de variados y efectivos sistemas de educación radiofónica le otorga a la comunicación y a los medios de masas una función relevante, (Kaplun, 1990) en el empleo del radio con las intenciones educativas en salud, sin embargo no ha llegado a significar un recurso que oportuna y adecuadamente sea utilizado por acciones programáticas de amplia cobertura y de carácter institucional (Horton, 2000; Schmelkes, 1971).

En el caso de México, son contadas las experiencias que se han reportado a cerca del empleo de la radio para estimular la organización de actividades educativas de beneficio comunitario (Sánchez, 1987). El uso de este medio, en buena medida, ha estado operando bajo la iniciativa de agrupaciones privadas, a las que mueven intereses no siempre libres de inspiraciones religiosas, políticas e ideológicas.

El pobre papel que tienen los medios de comunicación de masas en los planes y programas de educación y promoción higiénica debe sumarse al listado de los viejos problemas sanitarios. En todos los sectores de la actividad gubernamental, no solamente en el campo de la salud pública, la utilización del radio, de la televisión, de los periódicos y de las revistas invariablemente ha estado matizada por la falta de una voluntad coordinadora (Spangler, 1995; Strange, 1995) que utilice estos medios con fines sociales de desarrollo integral (Kozlowski et al., 1999).

Finalmente, el empleo del radio en la salud no se ha explorado en la totalidad de sus vertientes. De acuerdo a lo dicho, el sector salud en México ha visto en el medio electrónico un elemento publicitario, más que educativo. Para que el radio sea significativo como soporte de la educación sanitaria (Flora et al., 1989; Rees, 1999), las autoridades deberán preocuparse por investigar, diseñar, implementar y evaluar intervenciones futuras de comunicación educacional y vía radio que envuelvan en sus metodologías las estrategias nunca antes ensayadas en el plano institucional (Fortmann \& Varady, 2000; Strange, 1995; Verduin, 1994). 


\section{Referencias}

ALVA, S. A., 1995. Salud y Medios de Comunicación. México, DF: Editorial Conafe.

BITZER, O., 1993. Un Enfoque Andragógico. México, DF: Editorial Educar.

BOYD, N. R.; SUTTON, C.; ORLEANS, C. T.; McCLATCHEY, M. W.; BINGLER, R.; FLEISHER, L.; HELLER, D.; BAUM, S.; GRAVES, C. \& WARD, J. A., 1998. Quit today! A targeted communications campaign to increase use the cancer information service by African American smokers. Preventive Medicine, 27:S50-60.

DE ROUX, G. L., 1994. La Prevención de Comportamientos de Riesgo y la Promoción de Estilos de Vida Saludables en el Desarrollo de la Salud. Educación Medicina y Salud. México, DF: Editorial SEP.

DIAZ, N. L., 1993. Grupo de autocuidado en diabetes mellitus. Salud Pública de México, 35:169-176.

JAMISON, H. \& MCANANY, J., 1984. Radio instruccional. Chasqui, 10:38.

FLORA, J. A.; MAIBACH, E. W. \& MACCOBY, N., 1989. The role of media across four levels of health promotion intervention. Annual Review of Public Health, 10:181-201.

FORTMANN, S. P. \& VARADY, A. N., 2000. Effects of a community wide health education program on cardiovascular disease morbidity and mortality: The Stanford Five-City Project. American Journal of Epidemiology, 152:316-323.

HORTON, M., 2000. APHA offers new educational opportunities. American Journal of Public Health, 90:1193-1194

LINGFORS, H.; LINDSTROM, K.; PERSSON, L. G.; BENGTSSON, C. \& LISSNER, L., 2001. Evaluation of "Live for Life" a health promotion programme in the County of Skaraborg, Sweden. Journal of Epidemiology and Community Health, 55:277-282.

KAPLUN, M., 1990. El Comunicador Popular. Buenos Aires: Editorial Humanitas.

KOZLOWSKI, L. T.; GOLDBERG, M. E.; SWEENEY, C. T.; PALMER, R. F.; PILLITTERI, J. L.; YOST, B. A.; WHITE, E. L. \& STINE, M. M., 1999. Smoker reactions to a "radio message" that Light cigarettes: Dangerous as regular cigarettes. Nicotine \& Tobacco Research, 1:67-76.
MERCADO, R., 1990. Educación para la Salud. Madrid: Editorial Limusa.

NUÑEZ, H. C., 1989. Educar para Transformar, Comunicación, Educación y Cultura. Guadalajara: Editorial IMDEC.

REES, T., 1999. Radio talk can be valuable public relations tool. Profiles in Healthcare Marketing, 15:41.

ROGERS, E. M.; VAUGHAN, P. W.; SWALCHE, R. M.; RAO, N.; SVENKERUD, P. \& SOOD, S., 1999. Effects of an entertainment-education radio soap opera on family planning behavior in Tanzania. Studies in Family Planning, 30:193-211.

ROCHON, A., 1991. Educación para la Salud. Madrid: Editorial Masson.

SALLERAS, S., 1985. Educación Sanitaria. Madrid: Editorial Díaz de Santos.

SANCHEZ, R. E., 1987. Comunicación y Sociedad. Guadalajara: Editorial Universidad de Guadalajara.

SCHMELKES, S., 1971. Escuela Radiofónica Tarahumara. Revista del Centro de Estudios Educativos, 3:13-21.

SIEGEL, S., 1986. Estadística no Paramétrica Aplicada a las Ciencias de la Conducta. Madrid: Editorial Trillas.

SOLANO, S. M. A., 1992. Los Medios de Difusión de Masas. Guadalajara: Editorial Universidad de Guadalajara.

SPANGLER, A. N., 1995. Distant education and nutrition. Journal of the American Dietetic Association, 95:925-929.

STEINFATT, T. H., 1986. Comunicación Humana. México, DF: Editorial Diana.

STRANGE, J., 1995. Radio medical advice. Journal of the Royal Naval Medical Service, 81:12-15.

VAUGHAN, P. W.; ROGERS, E. M.; SINGHAL, A. \& SWALEHE, R. M., 2000. Entertainment education and HIV/AIDS prevention: A field experiment in Tanzania. Journal of Health Community, 5:81-100.

VERDUIN, J., 1994. Distant Education. Boston: Wisconsin.

VERHAEGEN, B., 1980. Educación, Cultura y Tecnología. México, DF: Editorial Nueva Imagen.

Recibido el 13 de marzo de 2000

Versión final presentada el 6 de noviembre de 2001

Aprobado el 5 de marzo de 2002 